\title{
Obstructive sleep apnea syndrome and sleep disorders in individuals with occupational injuries
}

\author{
Stig Solbach ${ }^{1}$, Katrin Uehli', Werner Strobel², Stefanie Brighenti-Zogg ${ }^{1}$, Selina Dürr ${ }^{1}$, Sabrina Maier ${ }^{1}$, Michel Hug $^{1}$, \\ Roland Bingisser ${ }^{3,4}$, Jörg Daniel Leuppi ${ }^{1,4}$ and David Miedinger ${ }^{1,4^{*}}$
}

\begin{abstract}
Background: Some sleep disorders are known risk factors for occupational injuries (Ols). This study aimed to compare the prevalence of obstructive sleep apnea syndrome (OSAS) in a population of patients with Ols admitted to the emergency room (ER) with hospital outpatients as controls.

Methods: Seventy-nine patients with Ols and 56 controls were recruited at the University Hospital of Basel, Switzerland between 2009 and 2011. All patients completed a questionnaire and underwent a full-night attended polysomnography (PSG). We considered an apnea-hypopnea index (AHI) $>5$ as an abnormal finding suggestive of a diagnosis of OSAS.

Results: Patients with Ols did not differ from controls regarding sex, age, body mass index, and job risk of Ol. Patients with Ols tended to have an abnormal AHI ( $n=38$ [48\%] vs. $n=16$ [29\%], odds ratio [OR] $=2.32$ [95\% confidence interval (CI):1.05-5.13]), and a higher AHI (8.0 vs. 5.6 events/h; Cohen's d 0.28, $p=0.028$ ) compared with controls. Patients with Ols also had abnormal limb movement index, arousal index, and signs of sleep bruxism compared with controls. Compared with 36 controls (66\%), 70 patients with Ols (89\%) had either excessive daytime sleepiness (EDS), and/or an abnormal finding during PSG (OR =4.32, 95\% Cl:1.65-11.52). However, patients with Ols did not differ from controls regarding EDS or oxygen desaturation index.

Conclusions: Patients treated in the ER for OI had more abnormal findings suggestive of OSAS or other sleep disorders compared with a control group of hospital outpatients. Screening for these conditions should be part of the postaccident medical investigation.
\end{abstract}

Keywords: Obstructive sleep apnea syndrome, Occupational accidents, Sleep fragmentation, Polysomnography, Excessive daytime sleepiness

\section{Background}

Obstructive sleep apnea syndrome (OSAS) is a respiratory sleep disorder with recurrent episodes of hypopnea, apnea, and associated arousals leading to fragmented sleep and, therefore, excessive daytime sleepiness (EDS) (Gharibeh and Mehra 2010). Studies in the general population of Switzerland reported a prevalence of $23.4 \%$ in women and 49.7\% in men (Heinzer et al. 2015). OSAS is a potential

\footnotetext{
* Correspondence: david.miedinger@unibas.ch

This study was performed at the University Hospital in Basel, Switzerland ${ }^{1}$ Cantonal Hospital Baselland, University Clinic of Medicine, $\mathrm{CH}-4410$ Liestal, Switzerland

${ }^{4}$ Medical Faculty, University of Basel, Basel, Switzerland

Full list of author information is available at the end of the article
}

risk factor for hypertension and cardiovascular disease and is associated with type 2 diabetes (Fava et al. 2011; Reichmuth et al. 2005). Studies have shown that salespersons, drivers, seamen, engine and motor operators, and cooks and stewards are at increased risk of being diagnosed with OSAS (Li et al. 2008).

The European Agency Eurostat defined an accident at work according to the European Statistics on Accidents at Work as "a discrete occurrence in the course of work which leads to physical or mental harm" (European Statistics on Accidents at Work (ESAW) 2013). In 2013, there were around 3.1 million nonfatal accidents in the European Union (EU-28), which led to an absence of at

(C) The Author(s). 2018 Open Access This article is distributed under the terms of the Creative Commons Attribution 4.0 International License (http://creativecommons.org/licenses/by/4.0/), which permits unrestricted use, distribution, and 
least 4 days from work and predominately affected male workers (Key figures on Europe 2016). We previously reviewed the published literature and estimated that 13\% of occupational injuries (OIs) could be attributed to sleep problems (Uehli et al. 2014; Uehli et al. 2013). Impaired self-reported sleep quality, sleep duration, and daytime sleepiness were significant risk factors for work injuries such as musculoskeletal injuries (Uehli et al. 2014; Uehli et al. 2013). OSAS and EDS can be considered as established risk factors for road traffic accidents and have been suggested to be important causes for injuries at the workplace (Garbarino et al. 2011; Arita et al. 2015; Akkoyunlu et al. 2013; Lindberg et al. 2001; Suzuki et al. 2005). However, the latter investigations were based on questionnaires about symptoms of OSAS, such as snoring and EDS, as well as self-reported or registered data on injuries occurring at work. It has been shown that OSAS screening questionnaires have a limited sensitivity and specificity of around 77 and 53\%, respectively, to diagnose OSAS in patients without history of sleep disorders (Abrishami et al. 2010). Retrospective assignment of injury status by questionnaire or consulting registries is prone to recall bias or underreporting.

In contrast, objective assessment using full-night attended polysomnography (PSG) - the gold standard for diagnosis of OSAS-might provide more reliable results at the time of injury. This would allow immediate counseling of the affected worker and quick initiation of evidence-based interventions to treat OSAS and EDS to decrease the risk for future and maybe more severe or even fatal OIs.

$\mathrm{n}$ this study, we investigated the prevalence of OSAS and other sleep disorders and symptoms of EDS in a sample of individuals who attended the emergency room (ER) of a tertiary hospital due to OI and compared them with a sample of hospital outpatients without recent history of injury at the workplace. Our hypothesis was that individuals with OI would have a higher prevalence of OSAS related sleep abnormalities in PSG than outpatients without OI.

\section{Methods}

We included patients aged between 17 and 65 years who attended the ER of the University Hospital of Basel, Switzerland, for treatment of a work-related injury (occupational injury patients [OIPs]). We enrolled males and females who fulfilled the following inclusion criteria: (1) aged between 16 and 65 years, (2) admission to hospital ER for a work-related injury on the day or subsequent day of injury, (3) moderate-to-serious injury severity (Grossmann et al. 2011), (4) sufficient German language skills, and (5) able to complete the questionnaire. During the same period, patients who were treated for acute conditions in the surgical outpatient department and who did not report workplace-related injuries in the last 3 months were recruited as controls (CONs). All participants were required to have a theoretical work capacity of $100 \%$ and to be employed at least $50 \%$ of the full-time equivalent.

All participants completed the Epworth Sleepiness Scale (ESS), a questionnaire to evaluate EDS (Johns 1991). We considered an ESS score of $>10$ points as indicative of EDS. Furthermore, we collected data related to the injury and the type of work they performed when injured and measured the patient's body weight and height. Work injury types were defined on the basis of groups of work injury variables that had been identified by factor analysis as published previously (Uehli et al. 2013). Patients' job risk was classified as "high risk" or "low risk" by a trained study nurse reading the relative work injury risk from the Swiss national accident statistics based on the respondent's primary job, age, and gender (Swiss National Accident Insurance Fund (Suva) 2009). The patient's job risk was classified as "high risk" if his relative work injury risk was greater than the 3 -year Swiss average.

Within 2 weeks after the OI, patients were investigated in the sleep laboratory. All subjects underwent a full-night attended PSG. A trained medical assistant monitored the patients and their recordings during the night to ensure continuous recording of data. We recorded chest and abdomen movements, nasal flow, pulse oximetry, electrocardiogram, submental and tibial electromyogram, electroencephalogram, and electrooculogram, accompanied by a microphone and infrared camera for monitoring purposes. Sleep data were analyzed by a trained physician qualified and experienced in the analysis of sleep studies, who was blinded with respect to group allocation of the patient (OIP or CON group). The PSG data were collected using RemLogic (Embla, Broomfield CO, USA). The AHI was calculated based on 2007 AASM Manual for Scoring Sleep and Associated Events. Hypopnea was defined as a reduction of airflow by at least $30 \%$ and followed by a drop in oxygen saturation of at least $4 \%$ from baseline for at least $10 \mathrm{~s}$. Apnea was defined as an airflow limitation of more than $90 \%$ of baseline. Sleep bruxism was assessed based on electromyogram activity. A cutoff of $>5$ was chosen to define an abnormal PSG result for the apnea-hypopnea index (AHI), oxygen desaturation index (ODI), limb movement index (LMI), and arousal index.

We used IBM SPSS Statistics version 24 for statistical analysis. Descriptive statistics were computed as mean and standard deviation. We used the Mann-Whitney $U$ test for continuous variables and Fisher's exact test for proportions. For continuous variables effect size expressed as Cohen's d was calculated. The Shapiro-Wilk test was used to examine whether data were normally distributed 
and histograms were assessed visually. The significance level was set at $p<0.05$.

\section{Results}

A total of 144 participants were enrolled (79 OIP, 66 $\mathrm{CON}$ ) and underwent a full-night attended PSG. However, we lost PSG data for 10 controls due to hard disk failure on the sleep laboratory computer. These individuals were excluded from further analysis. One individual in the OIP group was previously diagnosed with OSAS but refused treatment and was therefore included in the analysis.

Figure 1 shows the distribution of patients in the OIP and $\mathrm{CON}$ groups according to occupational categories of the International Standard Classification of Occupations, 1988. There was no difference between the OIP and CON groups regarding job risk for work-related injuries according to the Swiss National Accident Statistics 2007 edition (OIP $n=38$ [48\%] classified as "high risk" vs. CON $n=23$ [41\%] classified as "high risk," $p=0.484$ ). The proportion of females between groups did not differ significantly (OIP $n=32$ [41\%] vs. CON $n=29$ [53\%], $p=0.217$ ), and there was no difference in age (OIP $36 \pm 13$ years vs.
CON $39 \pm 13$ years; Cohen's $d-0.23, p=0.110$ ) or body mass index (BMI) (OIP $25.8 \pm 5.0 \mathrm{~kg} / \mathrm{m}^{2}$ vs. CON $24.7 \pm$ $4.12 \mathrm{~kg} / \mathrm{m}^{2}$; Cohen's d 0.24, $p=0.145$ ).

The results obtained by full-night attended PSG are presented in Table 1. The proportion of patients with an abnormal AHI as well as the mean AHI was higher in the OIP than the CON group. In contrast, there was no significant difference in the proportion of patients with an abnormal ODI or the mean ODI between the OIP and CON groups. Furthermore, patients in the OIP group comprised a higher proportion of individuals with signs of sleep bruxism and abnormal LMI and arousal index than the CON group.

Nine individuals each in the OIP and CON groups had an abnormal ESS score (equivalent to 11 and 16\% in the OIP and CON groups, respectively; OR 0.67 [95\% CI: $0.22-2.01])$. There was also no difference in mean ESS score between the OIP and CON groups (OIP 7.2 \pm 3.5 vs. CON $6.3 \pm 4.1$; Cohen's d 0.24, $p=0.190$ ).

We stratified patients in the OIP group according to AHI severity and saw that an increased AHI was associated with increasing age, BMI, and ESS and was present in higher proportion of males (Table 2).

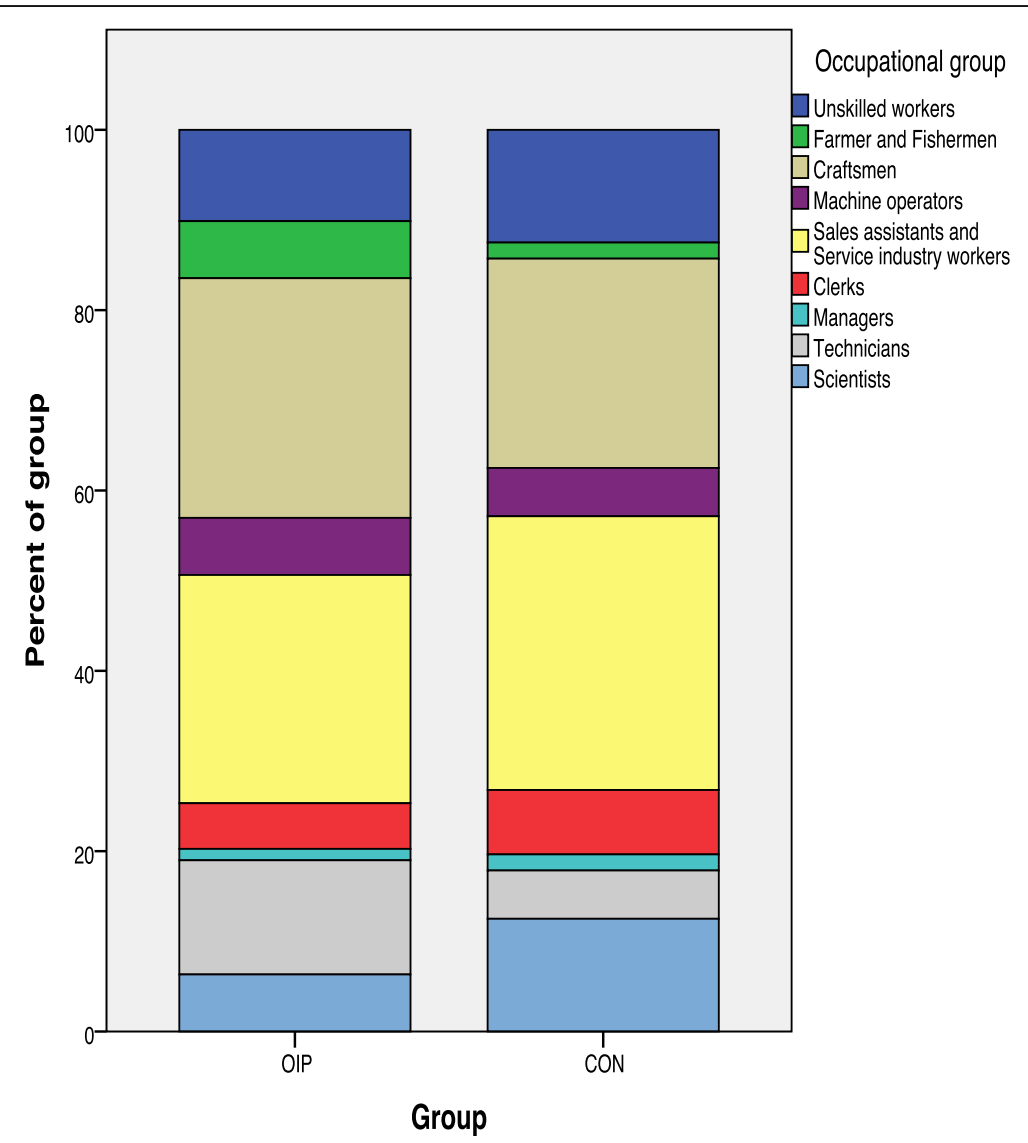

Fig. 1 Distribution of patients in the OIP and CON groups according to occupational categories of the International Standard Classification of Occupations (ISCO-88) (Bundesamt für Statistik 2017). Legend: OIP = occupational injuries patients, CON = controls 
Table 1 Individuals with excessive daytime sleepiness or abnormal findings during the sleep study with full-night attended polysomnography

\begin{tabular}{llll}
\hline & OIP group & CON group & \\
\hline Total sleep time (min) & $5.7( \pm 1.1)$ & $5.5( \pm 1.1)$ & Cohen's d 0.18, $p=0.210$ \\
AHI $>5(n)$ & $38(48 \%)$ & $16(29 \%)$ & OR 2.32 (1.05-5.13) \\
AHI (events/h) & $8.0( \pm 9.5)$ & $5.6( \pm 7.2)$ & Cohen's d 0.28, $p=0.028$ \\
ODI $>5(n)$ & $13(17 \%)$ & $9(16 \%)$ & OR 1.03 (0.37-2.87) \\
ODI (events/h) & $3.7( \pm 6.8)$ & $3.0( \pm 6.5)$ & Cohen's d 0.11, $p=0.698$ \\
LMI $>5(n)$ & $52(66 \%)$ & $22(40 \%)$ & OR 2.98 (1.38-6.46) \\
LMI (events/h) & $11.87( \pm 12.4)$ & $7.59( \pm 8.1)$ & Cohen's d 0.41, $p=0.030$ \\
Sleep bruxism $(n)$ & $8(10 \%)$ & $1(2 \%)$ & OR 6.20 (0.75-138.08) \\
Arousal index $>5(n)$ & $57(72 \%)$ & $28(51 \%)$ & OR 2.59 (1.19-5.67) \\
Arousal index (events/h) & $9.5( \pm 8.8)$ & $6.9( \pm 5.8)$ & Cohen's d 0.35, $p=0.011$ \\
Breathing-related arousals (events/h) & $6.0( \pm 6.6)$ & $4.4( \pm 5.7)$ & Cohen's d 0.26, $p=0.036$ \\
Unspecified arousals (events/h) & $2.7( \pm 1.6)$ & $2.6( \pm 1.6)$ & Cohen's d 0.06, $p=0.584$ \\
Abnormal PSG finding $(n)$ & $67(85 \%)$ & $35(64 \%)$ & OR 3.35 (1.38-8.25) \\
\hline
\end{tabular}

Data are presented as mean (SD) or frequency (\%) and odds ratio OR (95\% confidence interval (CI)) or effect size (Cohen's d) and p-value. Abnormal PSG finding was defined as at least one abnormal finding in the following categories: $\mathrm{AHI}>5, \mathrm{ODI}>5, \mathrm{LMI}>5$, sleep bruxism, arousal index $>5$

When considering the results of the ESS and the full-night attended PSG, 70 patients in the OIP group (89\%) and 36 controls (66\%) had either EDS and/or an abnormal finding (OR 4.32 95\% CI: 1.65-11.52). However, there was no difference in age, gender, BMI, and gender for individuals with or without EDS, respectively (age $39.9 \pm 14.9$ years vs. $37.3 \pm 12.1$ years; Cohen's d $0.19 p=0.964 ;$ BMI $24.6 \pm 4.0 \mathrm{~kg} / \mathrm{m}^{2}$ vs. $25.4 \pm 4.7 \mathrm{~kg} /$ $\mathrm{m}^{2}$; Cohen's d $-0.18 p=0.595$; 7 females (39\%) vs. $54 \mathrm{fe}-$ males (47\%), $p=0.617$ ).

Injury mechanism, type, location, and tasks during which work-related injuries occurred in the OIP group are shown in Table 3. Most of the patients were admitted for musculoskeletal injuries and wounds located at the extremities, and most injuries occurred while handling tools, machines, or loading and while moving at the workplace. The two most common injury mechanisms were stepping, caught/hit/crushed/struck by an object, or overloading.

\section{Discussion}

Individuals treated for OIs in the ER of a tertiary hospital in Switzerland and investigated with full-night attended PSG were found to have abnormal findings more frequently, such as elevated AHI and arousal index compatible with a diagnosis of OSAS compared with a control group of patients treated for other conditions in the same hospital. Furthermore, individuals with OIs were found to have a higher LMI and signs of sleep bruxism compared with controls. Our findings confirm previous studies suggesting that untreated OSAS and other sleep disorders need to be considered as important risk factors for injuries that occur at the workplace. Information about workers regarding symptoms and complications of OSAS together with PSG screening and subsequent treatment may offer an opportunity to decrease their risk of future injuries.

Our results support our primary hypothesis that an abnormal AHI is more frequent in patients with $\mathrm{OI}$ than in outpatients without OI. However, the differences in terms of prevalence of abnormal and mean AHI were relatively small and may be considered clinically insignificant. In post hoc analysis we compared several objective parameters obtained during the full-night attended PSG and found differences between patients with $\mathrm{OI}$ and without OI that were statistically significant

Table 2 Stratification of OIP group according to the apnea-hypopnea index (AHI)

\begin{tabular}{lllll}
\hline & AHI $<5(n=39)$ & AHI 5-9.9 $(n=21)$ & AHI 10-14.9 $(n=9)$ & AHI > 15 $(n=10)$ \\
\hline Age (years) & $30.05(9.7)$ & $38.38(12.3)$ & $45.11(14.0)$ & $44.1(12.7)$ \\
BMI $\left(\mathrm{kg} / \mathrm{m}^{2}\right)$ & $24.89(3.7)$ & $25.88(4.8)$ & $24.88(2.6)$ & $29.92(8.4)$ \\
ESS & $6.26(4.2)$ & $4.57(3.3)$ & $9.22(3.7)$ & $7.40(3.9)$ \\
Gender (males) & $21(53.8 \%)$ & $12(57.1 \%)$ & $6(66.7 \%)$ & $8(80 \%)$ \\
\hline
\end{tabular}

Data are presented as mean (SD) or frequency (\%) 
Table 3 Distribution of different work injury types according to apnea-hypopnea index (AHI) in the OIP group $(n=79)$

\begin{tabular}{llll}
\hline & $\mathrm{AHI}>5(n=38)$ & $\mathrm{AHI} \leq 5(n=41)$ & All OIP \\
\hline Caught, hit & 12 & 11 & 23 \\
Handling, carrying & 5 & 4 & 9 \\
Side task & 15 & 12 & 27 \\
Tool, machine & 13 & 13 & 26 \\
Object & 20 & 22 & 42 \\
Cut, open wound & 18 & 23 & 31 \\
Musculoskeletal injury, fall & 22 & 18 & 40 \\
Extremities & 37 & 37 & 74 \\
\hline
\end{tabular}

Data are presented as the number of individuals with a certain work injury type. "Caught, hit": Being caught, hit, crushed, or struck; "Handling, carrying": Handling or carrying loads by hand or using a handling device; "Side task": Carrying out a side task such as walking around, cleaning, tidying, changing clothes or taking a break; "Tool, machine": Involving a tool or machine, that is, for cutting, assembling, forming, lifting, or digging; "Object": Involving an object, that is, obstacles, inventory, ladders, or building parts; "Cut, open wound": Being cut or open wound; "Musculoskeletal injury, fall":

Musculoskeletal injury or slip, trip, or fall; "Extremities": Extremity, that is, hands, arms, legs, or feet

and would suggest that other sleep disorders may also be related with OI but the statistical significance did not pass Bonferroni correction for multiple testing. Therefore, these findings need to be confirmed in appropriately designed future studies.

OIs are caused by acute exposure to physical agents at the workplace, such as mechanical energy, electricity, chemicals and ionizing radiation, or by a sudden lack of essential agents, such as oxygen or heat. Injury events are caused by a complex interaction between factors associated with materials and equipment, the work environment, and the worker (Castillo et al. 2011). OIs represent a serious public health concern. It is estimated that every day approximately 1020 workers die as a result of OIs, and more than 960,000 workers are hurt because of accidents in the workplace (Hamalainen et al. 2009). Personal and occupational factors were found to be associated with an increased risk of OI and, as we have previously reported, approximately $13 \%$ of OIs can be attributed to sleep problems of any kind reflected by impaired sleep quality or quantity, breathing-related sleep problems, or the use of sleep medication (Uehli et al. 2014). Researchers analyzing data from the Canadian Community Health Survey (CCHS) found the strongest associations between work injury and sleep problems in men in trades and transportation jobs, women in processing and manufacturing jobs, and women who work rotating shifts (Kling et al. 2010).

Our sample of patients with OIs mainly consisted of individuals classified as craftsmen or sales assistants and service industry workers. According to the Swiss occupational health statistics male craftsmen of all ages and female craftsmen up to the age of 24 years have a high risk for OI.
The same is true for young male sales assistants and service industry workers (Swiss National Accident Insurance Fund (Suva) 2009). Our analysis did not reveal a systematic difference between OIP and CON regarding job risk for work-related injuries, proportion of males in the sample, age or BMI. We considered patients undergoing outpatient surgery as appropriate controls as they were 1) also engaged in regular employment and 2) were living and working in the hospitals catchment area and would therefore most likely have sought care for work-related injury in the same hospital.

In this study, individuals admitted with OIs were more frequently found to have findings compatible with a diagnosis of OSAS during sleep compared with the control group without OIs. These findings confirm previous research suggesting that OSAS might be a potential risk factor for injuries in the workplace. Ulfberg et al. investigated OI claims reported to the Occupational Injury Statistics register in Sweden in patients suffering from sleep-disordered breathing and in employed age-matched controls. They found that the risk of OI was increased in heavy snorers and patients with OSAS (Ulfberg et al. 2000). Similarly, Lindberg et al. reported an increased risk of OI in male snorers using questionnaires on snoring and EDS, as well as occupational accident reports to a national register (Lindberg et al. 2001). Acciantoli et al. studied workers with and without OSAS after full-night attended PSG and a questionnaire on self-judged work performance. Workers with OSAS reported to be affected by OI in the past more than those with normal sleep patterns (Accattoli et al. 2008). Furthermore, the authors stated that those with OSAS reported more difficulties with memory, impaired vigilance, concentration, ability to perform monotonous tasks, responsiveness, learning new tasks, and manual ability, and that the findings were associated with OSAS severity (Accattoli et al. 2008). Hassani et al. studied hospital workers in Iran using the Berlin questionnaire and data on self-reported OI. The workers classified as high risk for OSAS in the screening questionnaire were found to have a higher risk for self-reported accidents than those with a low-risk classification (Hassani et al. 2015). Finally, Hirsch and co-workers investigated patients with symptoms of OSAS referred to the sleep laboratory and reviewed occupational injury claims in the 5 years preceding the sleep study. They reported a twofold increase in the odds for OI in individuals with OSAS. Similar to our study, no consistent relationship between OI and OSA severity was found and EDS was not associated with OI (Hirsch Allen et al. 2016). Their study approach was different: The OI status was determined by using data obtained from the workers compensation board and only included OI cases that resulted in at least 1 day of absence from work and it is therefore possible that the actual number of $\mathrm{OI}$ is much higher when considering the fact that not all OI are reported and 
do not necessarily lead to lost workdays. Moreover, the interval between OI and the diagnosis of OSAS was much longer and the authors did not consider other abnormal sleep study findings that could be risk factors for OI.

In our study, we could not detect significant differences in the mean ODI and proportion of individuals with abnormal ODI. Our findings suggest that the cause of increased risk of $\mathrm{OI}$ is more attributable to fragmentation of sleep, rather than to recurrent severe desaturations. Factors affecting workers' arousal and attentiveness are known to be associated with an increased injury risk (Ulfberg et al. 2000). Fortunately, studies investigating drivers on a road safety platform, using a driving simulator and neuropsychological tests, have shown that impairments in vigilance, attention, and alertness seem to be partially reversible if individuals with OSAS undergo treatment with continuous positive airway pressure (CPAP). Mazza et al. showed that patients with OSAS with untreated OSAS had slower reaction times leading to a lengthening of vehicle stopping distance and an increased number of collisions compared with individuals without OSAS. These differences were no longer present after a 3-month period of CPAP treatment (Mazza et al. 2006). Orth et al. reported improvements in attention and alertness and a decrease in accident frequency and concentration faults in driving simulation and neuropsychological testing after initiation of CPAP treatment (Orth et al. 2005). As well as showing signs of immediate treatment success, there is evidence for an effect of CPAP treatment on road traffic accident rate (Yamamoto et al. 2000; Barbe et al. 2007). The use of oral appliances or hypoglossal stimulation therapy has not yet been shown to improve work performance or to reduce OI rate (Rabelo Guimaraes Mde and Hermont 2014). This emphasizes the importance of early detection and initiation of CPAP treatment to decrease the risk of OI (besides its well-known effect on cardiovascular risk reduction). Our findings confirm previously reported associations of age, BMI, and male gender with elevated AHI but not with ESS. This suggests that individuals with risk factors should be specifically targeted and counseled about OSAS and its associated adverse outcomes, even if they have not yet suffered an OI and do not report EDS.

We found a higher leg movement index in individuals with OI compared with controls. This finding either could reflect a reaction to breathing-related arousal or could be a result of underlying restless legs syndrome. Restless legs syndrome has been shown to disrupt sleep length, sleep quality, and daytime alertness and to impair quality of life and work productivity (Stevens 2015). A recent systematic review reported an association of sleep bruxism with sleep-related arousal, but a clear causative link with OSAS is still questionable (Jokubauskas and Baltrusaityte 2017). Until now, there has been no published evidence showing that individuals with restless legs syndrome or sleep bruxism are at higher risk of injuries. However, a recent study investigating firefighters with sleep questionnaires found that in individuals positively screened for restless legs syndrome, insomnia and shift work disorder had an increased risk of self-reported motor vehicle crashes, near crashes, nodding off or falling asleep while driving, and injuries in and out of work (Barger et al. 2015). Based on this, we suggest that patients with OIs should be screened for symptoms of restless legs syndrome and sleep bruxism, and that further studies are required to investigate the impact of these conditions on work safety outcomes.

Previous research has shown that workers with EDS have a more than twofold higher risk of sustaining an OI than workers without EDS (Melamed and Oksenberg 2002). The ESS questionnaire was designed to measure EDS but is frequently used in clinical practice-despite its limited diagnostic performance-to screen for OSAS (Osman et al. 1999). Our findings confirm that most individuals with only slightly abnormal AHI would probably not report EDS and that the ESS would therefore not be useful to identify these individuals. As our study results suggest, most would not be identified by nocturnal pulse oximetry either. Using only questionnaires to screen for OSAS has some limitations. Even specific screening questionnaires have only moderate diagnostic performance mainly due to their relatively low specificity (Abrishami et al. 2010; Ramachandran and Josephs 2009). Moreover, questionnaires, such as the ESS, have been shown to be prone to underreporting when used in the workplace setting (Parks et al. 2009). Therefore, authors have advocated screening for OSAS in commercial drivers using combined methods, such as data from questionnaires, physician-obtained history, BMI measurements, and functional tests and to complete the investigation with specific tests, such as portable monitors or full-night attended PSG (Kales and Straubel 2014).

We have previously reported an association between poor sleep quality, measured as low sleep efficiency, as well as a short sleep duration with injury during side tasks (Uehli et al. 2013). In the present analysis, the OIP group showed the most frequent work injury types involved an object (obstacles, inventory, ladders, or building parts), a musculoskeletal injury or slip, trip, or fall, or extremities (hands, arms, legs, or feet). However, the distribution of work injury type was similar in patients with abnormal and normal AHI measurements.

Our findings need to be interpreted with caution. We conducted our study in a hospital in the city center of Basel with nearby large production and service industries. The location of the hospital therefore probably attracted a high proportion of workers in industrial and service companies situated in the catchment area. Patients working in the primary sector (farming, fishing, 
foresting) were underrepresented in our study sample. Full-night attended PSG is an expensive diagnostic procedure and its availability is limited in many countries. However, studies have shown that portable monitors for home sleep testing in addition to clinical data (e.g., BMI) can be used to identify patients with OSAS without incurring greater costs (Gurubhagavatula et al. 2013). Eijsvogel et al. showed that a two-step screening with a questionnaire and a nasal flow recording was a promising way to screen for OSAS in a healthy working population (Eijsvogel et al. 2016). Administration of the questionnaire and the sleep investigation were carried out in the 2 weeks following the OI. We could not determine the impact of an OI on the outcome of the sleep investigation. Based on the available evidence from several studies, we believe that sleep-disordered breathing is the main cause of OI and that any reverse effect can probably be neglected. Although the use of opioid pain medication was not formal exclusion criteria, no individual in the OIP or CON group recorded taking such medication (data not shown).

We defined hypopneas according to the 2007 AASM recommended rules as a $30 \%$ reduction in airflow accompanied by a $4 \%$ oxygen desaturation. Applying AASM alternative scoring rules (a 50\% reduction in airflow accompanied by a $3 \%$ oxygen desaturation OR an arousal) may well have increased the difference in proportion of abnormal AHI and the mean AHI between individuals with occupational injuries and the controls. Thornton et al. have shown that using the AASM recommended scoring rules tends to yield lower mean and median AHI values compared to using the AASM alternative scoring rules (Thornton et al. 2012).

The analyzed study sample was rather small and decreased further due to data loss that occurred on our sleep laboratory computer. We lost data from six males and four females assigned to the CON group. Their mean BMI was similar, and their mean age was slightly higher than that of the analyzed individuals in the CON group (data not shown). In our sleep laboratory, we had limited capacity during weekends to investigate study participants. Therefore, we could only investigate patients who were able to undergo PSG during the weekend 2 weeks following injury. Therefore, while we cannot exclude a selection bias, we think that this would have affected OIP and CON groups in a similar fashion. Furthermore, the recruitment of study participants was limited to normal daytime working hours and our study sample mainly consisted of dayshift workers. Shift and night work are known to be associated with an increased OI risk (Wagstaff and Sigstad Lie 2011). We limited recruitment to patients with an emergency severity index of 3-4 and without trauma to the central nervous system or severe injury to the thoracic cage. Therefore, the observed associations need to be confirmed in future studies investigating patients with more severe OIs and other types of injury. Finally, we only included individuals with sufficient German language skills that allowed them to fill in the questionnaires used in this study.

\section{Conclusion}

In our study, individuals treated for OIs in the ER were shown to have more abnormal findings suggestive of OSAS or other sleep disorders compared with the control group of hospital outpatients. These results suggest that OSAS, as well as other sleep disorders, could be important risk factors for OI and therefore screening for these conditions should form part of the postaccident medical investigation. Furthermore, all individuals with known risk factors for OSAS such as male gender, higher age, and increased BMI should be counseled regarding OSAS, its adverse outcomes, and treatment options during regular health surveillance exams. Future studies are required to investigate the impact of OSAS screening and treatment on OI rate and how to approach high-risk workers with cost-effective diagnostic tests.

\section{Abbreviations \\ AHI: Apnea-hypopnea-index; BMI: Body-mass-index; Cl: Confidence intervall; CON: Controls; CPAP: Continous positive airway pressure; EDS: Excessive daytime sleepiness; ER: Emergency room; ESS: Epworth sleepiness scale; LMI: Limb movement index; ODI: Oxygen desaturation index; \\ OI: Occupational injuries; OIP: Occupational injuries patients; OR: Odds ratio; OSAS: Obstructive sleep apnea syndrome; p: p-value; PSG: Polysomnography; SD: Standard deviation}

\section{Acknowledgments}

The authors would like to thank all collaborating staff of the University Hospital of Basel, Switzerland, for their kind permission to recruit patients within their organization. We thank Dr. Werner Strobel for the analysis of the full-night attended PSG studies.

\section{Funding}

We gratefully acknowledge financial support from the Swiss National Accident Insurance Institution (Suva).

\section{Availability of data and materials}

The datasets used and analysed during the current study are available from the corresponding author on reasonable request.

\section{Authors' contributions}

$\mathrm{SS}, \mathrm{KU}, \mathrm{DM}$ and JDL were responsible for the study conception and design; DM, WS, RB, SD, SM, SB and JDL carried out the data acquisition; SS conducted the data analysis; SS drafted the manuscript; KU, DM, RB, SD, SM, $\mathrm{MH}, \mathrm{SB}$ and JDL critically revised the manuscript; and DM was responsible for primary supervision. All authors read and approved the final manuscript.

\section{Ethics approval and consent to participate}

The study protocol was approved by the local ethics committee (EKBB, trial registration number 37/09) on 02 April 2009. Participants were informed of the study objectives and gave their written informed consent prior to their inclusion.

\section{Competing interests}

David Miedinger and Katrin Uehli were employed by Suva during the data collection and analysis phase of this study. Other authors declare that they have no competing interests. 


\section{Publisher's Note}

Springer Nature remains neutral with regard to jurisdictional claims in published maps and institutional affiliations.

\section{Author details}

${ }^{1}$ Cantonal Hospital Baselland, University Clinic of Medicine, CH-4410 Liestal, Switzerland. ${ }^{2}$ Department of Pneumology, University Hospital of Basel, Basel, Switzerland. ${ }^{3}$ Department of Emergency Medicine, University Hospital of Basel, Basel, Switzerland. ${ }^{4}$ Medical Faculty, University of Basel, Basel, Switzerland.

\section{Received: 24 March 2018 Accepted: 29 May 2018}

Published online: 20 June 2018

\section{References}

Abrishami A, Khajehdehi A, Chung F. A systematic review of screening questionnaires for obstructive sleep apnea. Can J Anaesth. 2010;57(5):423-38.

Accattoli MP, Muzi G, dell'Omo M, et al. Occupational accidents, work performance and obstructive sleep apnea syndrome (OSAS). G Ital Med Lav Ergon. 2008:30(3):297-303.

Akkoyunlu ME, Altin R, Kart L, et al. Investigation of obstructive sleep apnoea syndrome prevalence among long-distance drivers from Zonguldak, Turkey. Multidiscip Respir Med. 2013;8(1):10.

Arita A, Sasanabe R, Hasegawa R, et al. Risk factors for automobile accidents caused by falling asleep while driving in obstructive sleep apnea syndrome. Sleep Breath. 2015:19(4):1229-34.

Barbe F, Sunyer J, de la Pena A, et al. Effect of continuous positive airway pressure on the risk of road accidents in sleep apnea patients. Respiration. 2007;74(1):44-9.

Barger LK, Rajaratnam SM, Wang W, et al. Common sleep disorders increase risk of motor vehicle crashes and adverse health outcomes in firefighters. J Clin Sleep Med. 2015;11(3):233-40.

Bundesamt für Statistik. International Standard Classification of Occupations ISCO 88 (COM). 2017. https://www.bfs.admin.ch/bfs/de/home/statistiken/ arbeit-erwerb/nomenclaturen/isco-88-com.html, Accessed 23 Mar 2018.

Castillo DN, Pizatella TJ, Stout NA. Injuries and Occupational Safety. In: Levy BS, Wegman DH, Baron SL, Sokas RK, editors. Occupational and envrionmental health - recognizing and preventing disease and injury. 6th ed. New York: Oxford University Press; 2011. p. 315-34.

Eijsvogel MM, Wiegersma S, Randerath W, Verbraecken J, Wegter-Hilbers E, van der Palen J. Obstructive sleep apnea syndrome in company workers: development of a two-step screening strategy with a new questionnaire. J Clin Sleep Med. 2016;12(4):555-64.

European Statistics on Accidents at Work (ESAW) - Summary methodology. 2013th. Luxembourg: Publications Office of the European Union; 2013. http:// ec.europa.eu/eurostat/en/web/products-manuals-and-guidelines/-/KS-RA-12102. Accessed 13 Mar 2018.

Fava C, Montagnana M, Favaloro EJ, Guidi GC, Lippi G. Obstructive sleep apnea syndrome and cardiovascular diseases. Semin Thromb Hemost. 2011;37(3): 280-97.

Garbarino S, Traversa F, Spigno F, Bonsignore AD. Sleepiness, sleep disorders and risk of occupational accidents. G Ital Med Lav Ergon. 2011;33(3 Suppl):207-11.

Gharibeh T, Mehra R. Obstructive sleep apnea syndrome: natural history, diagnosis, and emerging treatment options. Nat Sci Sleep. 2010;2:233-55.

Grossmann FF, Nickel $\mathrm{CH}$, Christ M, Schneider K, Spirig R, Bingisser R. Transporting clinical tools to new settings: cultural adaptation and validation of the emergency severity index in German. Ann Emerg Med. 2011;57(3): 257-64.

Gurubhagavatula I, Fields BG, Morales CR, et al. Screening for severe obstructive sleep apnea syndrome in hypertensive outpatients. J Clin Hypertens (Greenwich). 2013:15(4):279-88.

Hamalainen P, Leena Saarela K, Takala J. Global trend according to estimated number of occupational accidents and fatal work-related diseases at region and country level. J Saf Res. 2009;40(2):125-39.

Hassani S, Rahnama N, Seyedmehdi SM, et al. Association between occupational accidents and sleep apnea in hospital staff. Tanaffos. 2015:14(3):201-7.

Heinzer R, Vat S, Marques-Vidal P, et al. Prevalence of sleep-disordered breathing in the general population: the HypnoLaus study. Lancet Respir Med. 2015; 3(4):310-8

Hirsch Allen AJ, et al. Obstructive sleep apnoea and frequency of occupational injury. Thorax. 2016;71:664-6.
Johns MW. A new method for measuring daytime sleepiness: the Epworth sleepiness scale. Sleep. 1991:14(6):540-5.

Jokubauskas L, Baltrusaityte A. Relationship between obstructive sleep apnoea syndrome and sleep bruxism: a systematic review. J Oral Rehabil. 2017:44(2): 144-53.

Kales SN, Straubel MG. Obstructive sleep apnea in north American commercial drivers. Ind Health 2014:52(1):13-24.

Key figures on Europe. Luxembourg: eurostat; 2016. http://ec.europa.eu/eurostat/ web/products-statistical-books/-/KS-El-16-001. Accessed 13 Mar 2018.

Kling RN, McLeod CB, Koehoorn M. Sleep problems and workplace injuries in Canada. Sleep. 2010:33(5):611-8.

Li X, Sundquist K, Sundquist J. Socioeconomic status and occupation as risk factors for obstructive sleep apnea in Sweden: a population-based study. Sleep Med. 2008;9(2):129-36.

Lindberg E, Carter N, Gislason T, Janson C. Role of snoring and daytime sleepiness in occupational accidents. Am J Respir Crit Care Med. 2001; 164(11):2031-5.

Mazza S, Pepin JL, Naegele B, et al. Driving ability in sleep apnoea patients before and after CPAP treatment: evaluation on a road safety platform. Eur RespirJ. 2006:28(5):1020-8.

Melamed S, Oksenberg A. Excessive daytime sleepiness and risk of occupational injuries in non-shift daytime workers. Sleep. 2002;25(3):315-22.

Orth M, Duchna HW, Leidag M, et al. Driving simulator and neuropsychological [corrected] testing in OSAS before and under CPAP therapy. Eur Respir J. 2005;26(5):898-903

Osman EZ, Osborne J, Hill PD, Lee BW. The Epworth sleepiness scale: can it be used for sleep apnoea screening among snorers? Clin Otolaryngol Allied Sci. 1999;24(3):239-41.

Parks P, Durand G, Tsismenakis AJ, Vela-Bueno A, Kales S. Screening for obstructive sleep apnea during commercial driver medical examinations. J Occup Environ Med. 2009:51(3):275-82

Rabelo Guimaraes Mde L, Hermont AP. Sleep apnea and occupational accidents: Are oral appliances the solution? Indian J Occup Environ Med. 2014;18(2):39-47.

Ramachandran SK, Josephs LA. A meta-analysis of clinical screening tests for obstructive sleep apnea. Anesthesiology. 2009:110(4):928-39.

Reichmuth KJ, Austin D, Skatrud JB, Young T. Association of sleep apnea and type II diabetes: a population-based study. Am J Respir Crit Care Med. 2005; 172(12):1590-5

Stevens MS. Restless legs syndrome/Willis-Ekbom disease morbidity: burden, quality of life, cardiovascular aspects, and sleep. Sleep Med Clin. 2015;10(3): 369-73. xv-xvi

Suzuki K, Ohida T, Kaneita Y, Yokoyama E, Uchiyama M. Daytime sleepiness, sleep habits and occupational accidents among hospital nurses. J Adv Nurs. 2005; 52(4):445-53.

Swiss National Accident Insurance Fund (Suva). Accident Statistics (Federal Law on Accident Insurance (UVG)) 2003-2007. 2009.

Thornton AT, Singh P, Ruehland WR, Rochford PD. AASM Criteria for scoring respiratory events: interaction between apnea sensor and hypopnea definition. Sleep. 2012;35(3):425-32. https://doi.org/10.5665/sleep.1710.

Uehli K, Mehta AJ, Miedinger D, et al. Sleep problems and work injuries: a systematic review and meta-analysis. Sleep Med Rev. 2014;18(1):61-73.

Uehli K, Miedinger D, Bingisser R, et al. Sleep problems and work injury types: a study of 180 patients in a Swiss emergency department. Swiss Med Wkly. 2013;143:w13902

Ulfberg J, Carter N, Edling C. Sleep-disordered breathing and occupational accidents. Scand J Work Environ Health. 2000;26(3):237-42

Wagstaff AS, Sigstad Lie JA. Shift and night work and long working hours-a systematic review of safety implications. Scand J Work Environ Health. 2011; 37(3):173-85.

Yamamoto H, Akashiba T, Kosaka N, Ito D, Horie T. Long-term effects nasal continuous positive airway pressure on daytime sleepiness, mood and traffic accidents in patients with obstructive sleep apnoea. Respir Med. 2000;94(1): 87-90. 